\title{
EDITORIAL
}

\section{PAISAJES, PATRIMONIO Y GOBERNANZA TERRITORIAL DE LOS SISTEMAS AGROALIMENTARIOS LOCALES}

\author{
Javier Sanz Cañada \\ Coordinador del grupo de investigación "Sistemas Agroalimentarios y Desarrollo Territorial" \\ del Instituto de Economía, Geografía y Demografía (IEGD / CSIC) \\ javier.sanz@cchs.csic.es \\ ORCID iD: https://orcid.org/0000-0002-2404-9488
}

Tomás García Azcárate

Vicedirector del Instituto de Economía, Geografía y Demografía (IEGD / CSIC)

tomas.gazcarate@cchs.csic.es

ORCID iD: https://orcid.org/0000-0003-2902-886X

Durante las últimas décadas, la literatura científica internacional ha incorporado a los análisis de Geografía Agroalimentaria enfoques de carácter socioeconómico, político y cultural que inciden en la investigación de las interconexiones que los sistemas agroalimentarios locales (SIAL) tienen con el territorio en el que se ubican. Frente al predominio de una agricultura y una alimentación globales que tienden a desproveerse de sus particularidades territoriales, otros tipos de sistemas agroalimentarios locales y/o alternativos manifiestan intensas interconexiones con el territorio y una singularidad espacial. En la intersección entre las investigaciones geográficas y los análisis de cadenas agroalimentarias, diferentes escuelas de pensamiento han estudiado las interrelaciones causales entre el territorio y los sistemas locales de producción de alimentos, entre las que podemos citar a las escuelas anglosajonas de Geografía Agroalimentaria denominadas Alternative Agro-food Networks, a los enfoques de Agroecología y a las escuelas, de origen franco-mediterráneo, sobre Systèmes Agro-alimentaires Localisés (Sistemas Agroalimentarios Locales, SIAL).

En este número especial de la Revista de Estudios Geográficos pretendemos ofrecer una muestra representativa de la investigación sobre las interrelaciones que tienen lugar entre los paisajes y el patrimonio agroalimentario, por una parte, y las construcciones sociales que a lo largo del tiempo construyen, patrimonializan, conservan y destruyen dichos paisajes, por otro, mediante el concurso de diferentes modelos de gobernanza territorial. El ámbito de estudio es el local y el objeto de estudio son los sistemas agroalimentarios. Una selección de los trabajos presentados al XII Congreso Iberoamericano de Estudios Rurales (CIER XII, Segovia, 2018) que versaban sobre estos temas, ha alimentado una parte significativa de los artículos que finalmente han sido seleccionados y evaluados.

Este número especial está estructurado en tres partes que abordan, desde distintos ángulos multidisciplinarios complementarios, la problemática del paisaje, el patrimonio y la gobernanza territorial de los SIAL en territorios rurales, rururbanos y urbanos. Una primera parte hace especial énfasis en el análisis a las relaciones existentes 
entre paisajes y patrimonio agroalimentario. En primer lugar, el concepto de paisaje implica específicamente la existencia de una percepción social de la población sobre el territorio, es decir, sobre los factores físicos y humanos que, fruto de su interrelación a lo largo del tiempo, conforman sus características diferenciales. Es así que el paisaje depende de la interacción entre percepción y dimensión material (Droeven et al., 2007) ${ }^{1}$. Cuando las poblaciones locales perciben que el paisaje es utilizable es cuando adquiere la dimensión de recurso (Zubelzu y Allende, $2015)^{2}$ y, más en particular, de recurso patrimonial: ello sucede cuando la sociedad logra un consenso en otorgar un valor a un paisaje determinado y dotarle de mecanismos de protección. En segundo término, la patrimonialización de los productos agrarios y alimentarios se traduce en un proceso histórico cultural de construcción de referencias identitarias comunes a escala local. Su objetivo es conferir a los alimentos un mayor significado simbólico y una mayor densidad cultural (Sanz-Cañada y Muchnik, 2016) ${ }^{3}$. Entre estos significados y referencias culturales de los alimentos, los paisajes donde son producidos ocupan un rol protagonista.

En consecuencia, esta percepción social inherente al concepto de paisaje es susceptible de ser empleada en la elaboración de un discurso y una narrativa destinados a emprender un proceso de patrimonialización de los paisajes agroalimentarios y de sus alimentos identitarios y, por tanto, de su conservación y su puesta en valor. En el presente número de Estudios Geográficos aportamos cuatro trabajos que abordan esta temática: tres sobre paisajes vitivinícolas en Castilla y León, que responden a tipologías espaciales bien distintas, y un artículo sobre patrimonialización de salinas históricas de interior.

Molinero Hernando y Martínez Arnáiz, en su trabajo sobre "Nuevos paisajes y nuevas formas de producción: la expansión de las empresas de servicios y de los asalariados en los espacios vitícolas del Duero", investigan los cambios en el paisaje que están ocurriendo con cierta celeridad en determinadas denominaciones de origen protegidas (DOP) vitivinícolas del valle y las campiñas del Duero (Rueda, Ribera, Toro...). Estas DOP se caracterizan por haber tenido durante los últimos años una importante expansión de su viñedo, así como una creciente reputación y un incremento sensible en la demanda de sus vinos. Asimismo, ha tenido lugar una inversión considerable en nuevas plantaciones más intensivas (en espaldera) y de mayor tamaño, que responden a criterios de re- ducción significativa de costes de producción. La gran novedad es que ha surgido una reorganización del trabajo en la vitivinicultura para que pequeños productores de edad media avanzada, fraccionados en numerosas parcelas, puedan aprovechar las ventajas derivadas de un aumento en el conocimiento y aprecio de sus vinos por parte del consumidor. Es así que ha surgido una red de empresas de servicios, que son cooperativas laborales de trabajadores (en su mayoría emigrantes), en las que los viticultores han externalizado numerosas tareas del cultivo de la vid e incluso de su administración y gestión. Esta nueva viticultura intensiva ha tenido como mérito contribuir en muchas zonas a combatir la despoblación, pero ha ocasionado también una homogeneización paisajística incuestionable, que se concreta visualmente en el creciente predominio del viñedo en espaldera.

Frente a estos procesos de intensificación que tienden a una mayor homogeneización del paisaje, Baraja Rodríguez y Herrero Luque investigan otro tipo de procesos de patrimonizalización de DOP vitivinícolas, vinculados a parajes con grandes potencialidades paisajísticas y turísticas: las DOP de Sierra de Francia, Cebreros, el Bierzo y los Arribes del Duero. En su artículo "Crisis y pervivencia de los paisajes vitivinícolas en los bordes de Castilla y León: dinámica, estrategias e instrumentos contra el abandono y la pérdida de valores patrimoniales", los autores analizan el impacto de las iniciativas cuya finalidad ha sido corregir el abandono y el deterioro de los valores paisajísticos y patrimoniales de los viñedos. Tras constatar que las medidas aplicadas han tenido resultados marcadamente desiguales, los autores concluyen que la recomposición de los lazos con el territorio se convierte así en el factor determinante de la calidad ambiental y cultural de los terroir vitivinícolas.

En el tercer artículo, titulado "Valor patrimonial de los paisajes del vino y su promoción en los valles y llanuras del este de Zamora", Plaza Gutiérrez y Fernández Álvarez hacen también una apuesta decidida por la recuperación y gestión del patrimonio como pieza clave de una estrategia de desarrollo territorial que conjuga producción vinícola y enoturismo. Los autores analizan comparativamente el valor patrimonial los paisajes vitivinícolas de tres DOP del Este de Zamora -Toro, Tierra del Vino y Valles de Benaventemediante la identificación de los recursos que ofrecen los tres territorios y mediante el análisis del discurso que guía sus respectivas estrategias de promoción.

Las salinas tradicionales de interior son un ejemplo de estrategias de patrimonialización que cuentan con 
un importante patrimonio arquitectónico y cultural que, sin embargo, ha abandonado su función productiva, que requiere ser rescatada. Hueso Kortekaas, en su artículo "La patrimonialización de las salinas tradicionales: una herramienta para el desarrollo local", compara la evolución y la situación de nueve salinas españolas, con los casos de tres procesos de patrimonialización exitosos en Francia (salinas de Guérande), Eslovenia (salinas Seĉovje) y Dinamarca (salinas de Læsø). Los casos de éxito comparten el hecho de haber albergado amplios y transparentes procesos de participación de las sociedades locales en la patrimonialización de sus salinas. Con el fin de conseguir empoderar a las sociedades locales, así como de crear identidad y sentido de pertenencia, pueden ser de utilidad tanto la organización de actividades educativas, científicas, artísticas, deportivas y sociales como las alianzas con las empresas y las asociaciones locales.

La segunda y la tercera parte del número monográfico hacen referencia al análisis de los procesos de gobernanza local y territorial en los SIAL. LoS productores agrarios y agroindustriales, las empresas de servicios, los distribuidores y las instituciones locales, pueden generar sinergias locales, fruto de las relaciones no sólo de proximidad geográfica sino también de proximidad organizativa (Torre y Talbot, $2018)^{4}$, en las que agentes e instituciones locales comparten redes de relaciones y sistemas de valores (sostenibilidad de la producción agroalimentaria, consumo preferente en circuitos cortos, etc.). Entendemos que la gobernanza territorial implica el desarrollo de procesos de organización colectiva en red donde tienen lugar a nivel local interacciones de distinto tipo (de compra-venta, de difusión de la información, de innovación, etc.) entre los agentes, las empresas y las instituciones, en las que intervienen diferentes procesos de coordinación multi-nivel (Torre y Traversac, 2011)5. La difusión de innovación y conocimientos en el ámbito de las redes locales adquiere un rol central en estos procesos. No existen vías únicas para el éxito en las trayectorias de gobernanza territorial, por lo que en el presente special issue sólo podemos exponer una pequeña muestra de la gran variedad de respuestas geográficas a la conformación de las redes locales y a la solución de los problemas de gobernanza de los SIAL.

La segunda parte del número monográfico está dedicada a exponer modelos de gobernanza territorial en ámbitos rurales, donde mostramos diferentes tipos de institucionalidades relacionadas con la go- bernanza de los bosques, con la gobernanza agroalimentaria y, dentro de esta última, con la gobernanza agroecológica en modelos de custodia del territorio.

Cruz y García-Bengochea exponen en su artículo "Vínculos socio-espaciales y gobernanza local: apego al lugar y participación en la iniciativa Bosque Modelo Palencia" una sugerente iniciativa de gobernanza forestal, impulsada en 2017 mediante un modelo asociativo basado en el arraigo de la población local. Las autoras obtienen una conclusión de interés para los estudios de gobernanza territorial en SIAL: observan que las personas más implicadas en este tipo de iniciativas presentan fuertes vínculos con el lugar, que se retroalimentan cuando acaecen dinámicas colectivas de desarrollo local. Dichos vínculos de arraigo refuerzan las valoraciones positivas de la ruralidad y refuerzan la decisión de permanecer en el territorio. Las dinámicas colectivas tienen entre sus finalidades trabajar los procesos subjetivos que refuerzan el arraigo.

Torres Salcido, Sandoval Moreno y Burbano Muñoz, en su artículo "Análisis de dos modelos de gobernanza: los sistemas agroalimentarios locales de zarzamora en México y de mora en Colombia", realizan un análisis comparativo de dos modelos de gobernanza del SIAL de frutas del bosque con rasgos muy distintos, tanto por su respectiva orientación comercial como por su localización geográfica: el caso mexicano corresponde a un cultivo de exportación, mientras que el colombiano a un cultivo propio de la economía campesina local. Ambos casos responden a un modelo de gobernanza local de carácter jerárquico en la toma de decisiones, dominado por los principales exportadores en el primer caso y por la gestión pública en el segundo. Sin embargo, resulta interesante observar que, en los intersticios del modelo dominante, se construyen de forma incipiente en ambos SIAL formas de gobernanza descentralizada basadas en la difusión del conocimiento y el arraigo territorial del producto.

Yacamán-Ochoa y García-Llorente, en su artículo titulado "Enfoque cooperativo y custodia del territorio: dos factores impulsores de la transición agroecológica de los sistemas agroalimentarios locales", investigan los factores impulsores de la transición agroecológica en SIAL que han adoptado iniciativas de "custodia del territorio", en dos ámbitos geográficos de estudio: la asociación Tierra Sana, que agrupa a productores agroecológicos del Valle del Jerte, y el Foro "Asturias Sostenible", que es una red de agricul- 
tura y ganadería extensiva y sostenible en paisajes de montaña de Asturias. Las autoras demuestran que las redes colaborativas enraizadas en el territorio, basadas en acuerdos colectivos voluntarios de colaboración a escala local entre agentes pertenecientes a distintas fases de la cadena alimentaria, resultan ser factores clave para promover una gobernanza territorial inclusiva. La escala local-regional favorece la creación de alianzas estructurales entre los agentes como resultado de la interacción social y la proximidad espacial, lo que permite a los pequeños productores mejorar su participación y empoderamiento en los SIAL. El artículo verifica afirmativamente la hipótesis de que las estructuras de autogobierno enraizadas en el territorio, que se organizan para gestionar sus propios recursos según sus propios valores y normas, juegan un papel esencial para generar sinergias colectivas beneficiosas.

La tercera parte del presente número de la revista versa principalmente sobre los modelos de gobernanza territorial alimentaria que han surgido en tiempos recientes a iniciativa de las ciudades, sobre todo a raíz de la firma del Pacto de Milán en 2015, lo que tiene grandes implicaciones en las áreas rururbanas y rurales circundantes. La densa red de interacciones inherente a los SIAL vinculados a la alimentación sostenible, se hace más compleja cuando tienen lugar saltos de escala en la producción y en el consumo. Los artículos que reseñamos a continuación reflejan también la existencia de una gran variedad de obstáculos a remontar cuando se amplía la escala, que son no sólo logísticos y de índole material, sino también de carácter cultural. Así, la construcción de confianza, el aprender a trabajar juntos, el comprender que todos los actores tienen mucho que ganar con las estrategias colaborativas, son procesos de "cocción lenta", por implicar cambios profundos de mentalidad y de actuación, pero imprescindibles a la hora de asegurar la durabilidad de las iniciativas asociativas innovadoras.

López-García, Alonso-Leal, García-García, MoleroCortés, García-Fernández, Arroyo-Escudero y HerreraCalvo, de la Fundación Entretantos, aportan una interesante contribución teórica y empírica sobre tipologías de modos de gobernanza local que tienen lugar en las estrategias alimentarias de las ciudades: "Ámbitos de gobernanza en las políticas alimentarias urbanas: una mirada operativa". Emplean, como base empírica, las medidas emprendidas para mejorar la gobernanza alimentaria en trece ciudades españolas. Los distintos tipos de gobernanza que inciden en las políticas alimentarias urbanas de corte participativo son, según los autores, las siguientes: gobernanza de base; multi-actor; intra-administración; multi-nivel; territorial y translocal. En torno a cada uno de estos ámbitos de gobernanza, se crean nuevas institucionalidades multi-actor, que tienen como misión posibilitar la dialéctica entre la administración, el sector agroalimentario, las organizaciones sociales y los consumidores.

Oñederra Aramendi, Begiristain Zubillaga y Malagón Zaldúa, en su trabajo sobre "El centro de acopio Sareko: aprendizajes para la gobernanza de las redes agroalimentarias alternativas en un salto de escala", analizan la gobernanza de nuevas fórmulas asociativas vinculadas a la alimentación sostenible que presentan marcados rasgos de innovación social: los centros de acopio (centros logísticos o food hubs) de productores agroecológicos cuyos alimentos se destinan a la restauración comercial. El centro de acopio Sareko tiene como finalidad abastecer a restaurantes de la comarca de Donostialdea, en Gipuzkoa, en torno a la ciudad de Donostia. Las autoras parten de la premisa de que la logística de los pequeños productores constituye uno de los grandes obstáculos al salto de escala en las redes agroalimentarias alternativas. EI artículo demuestra que Sareko responde al concepto de innovación social, no sólo por contribuir al fomento tanto de la transición ecológica (productoras agroecológicas) como de la transformación social (economía social y solidaria, gobernanza ascendente), sino también por la gran novedad que supone la creación de centros logísticos agroecológicos especializados en la restauración comercial. El trabajo concluye que para lograr el salto de escala en agroecología hay que apoyarse en iniciativas que hibriden la comunicación, la negociación y la construcción de confianza entre los agentes participantes en las redes

Finalmente, en la Nota sobre "La compra pública alimentaria en la regeneración agroecológica del paisaje periurbano de la ciudad de Madrid", cuyas autoras son Simón-Rojo, Morán y del Valle, se calcula el potencial transformador que podría tener en la ciudad de Madrid una política de compra pública dirigida al sector agroecológico. Se realiza un cálculo realista, sólo para las frutas y las legumbres cultivadas en la Comunidad de Madrid, basado en una ampliación del programa de ecocomedores de las escuelas infantiles municipales. A pesar de que el programa es de una magnitud mínima para una ciudad del tamaño de Madrid, la producción agroecológica regional dista mucho de ser suficiente para abastecer di- 
chos comedores públicos. Es decir, un mínimo impulso a las políticas de compra pública requeriría en Madrid poner en marcha de forma sincronizada políticas de impulso a la producción y de fomento a la logística agroecológica.

Tras la irrupción de la pandemia de la Covid 19 ha incrementado en nuestra sociedad la propensión a demandar una alimentación más saludable, más respetuosa del medio ambiente y producida más localmente, lo que aparece confrontado con el hecho de que los consumidores comienzan a tener menores disponibilidades económicas a causa de la crisis económica resultante de la crisis sanitaria. En este con-

\section{NOTAS}

1 Droeven, E., Dubois, C. y Feltz, C. (2007). Paysages patrimoniaux en Wallonie (Belgique), analyse par approche des paysages témoins. Cahiers d'Économie et Sociologie Rurales, 84-85, 215-243.

2 Zubelzu Mínguez, S. y Allende Álvarez, F. (2015). El concepto de paisaje y sus elementos constituyentes: requisitos para la adecuada gestión del recurso y adaptación de los instrumentos legales en España. Cuadernos de Geografía / Revista Colombiana de Geografía, 24 (1), 29-42. doi: 10.15446/rcdg.v24n1.41369 texto, la resolución de los desafíos que afrontará la alimentación sostenible en el futuro próximo requiere estrechar lazos entre las ciudades y sus entornos rururbanos y rurales, así como cooperar en la creación de redes interdisciplinares de innovación y acción, en el ámbito de economías más territorializadas y menos globalizadas. Emprender procesos de gobernanza territorial participativa que patrimonialicen los alimentos y los paisajes agroalimentarios que los albergan, parece constituir al menos un punto de partida para la solución a estos dilemas. Esperamos que el presente número especial de Estudios Geográficos aporte luz a este apasionante debate.

3 Sanz-Cañada, J. y Muchnik, J. (2016). Geographies of Origin and Proximity: approaches to Local Agrofood Systems. Culture \& History, 5 (1): e002. doi:10.3989/chdj.2016.002

4 Torre, A. y Talbot D. (2018). Proximités : retour sur 25 années d'analyse, Revue d'Économie Régionale \& Urbaine, 2018/5-6, 917-936. doi:10.3917/ reru.185.0917

5 Torre, A. y Traversac, J.B. (eds.) (2011). Territorial Governance. Local Development, Rural Areas and Agrofood Systems. New York, USA: Springer. ISBN 978-3-7908-2950-1. 